\title{
A Randomized Phase 1 Safety, Pharmacokinetic and Pharmacodynamic Study of the Novel Myostatin Inhibitor Apitegromab (SRK-015): A Potential Treatment for Spinal Muscular Atrophy
}

\author{
Doreen Barrett · Sanela Bilic · Yung Chyung · Shaun M. Cote · \\ Ryan Iarrobino · Katherine Kacena · Ashish Kalra · Kimberly Long • \\ George Nomikos (D) - Amy Place · James Gordon Still · Leela Vrishabhendra
}

Received: March 15, 2021 / Accepted: April 20, 2021 / Published online: May 8, 2021

(c) The Author(s) 2021

\section{ABSTRACT}

Introduction: Apitegromab (SRK-015) is an anti-promyostatin monoclonal antibody under development to improve motor function in patients with spinal muscular atrophy, a rare neuromuscular disease. This phase 1 doubleblind, placebo-controlled study assessed safety, pharmacokinetic parameters, pharmacodynamics (serum latent myostatin), and immunogenicity of single and multiple ascending doses of apitegromab in healthy adult subjects.

Methods: Subjects were administered single intravenous ascending doses of apitegromab of $1,3,10,20,30 \mathrm{mg} / \mathrm{kg}$ or placebo, and multiple

D. Barrett · Y. Chyung - S. M. Cote - R. Iarrobino . A. Kalra · K. Long · G. Nomikos $(\bowtie) \cdot$ A. Place Scholar Rock, Inc., 301 Binney Street, 3rd Floor, Cambridge, MA 02142, USA

e-mail: gnomikos@scholarrock.com

S. Bilic

Vanadro, LLC, Urbandale, IA, USA

K. Kacena

Katherine Kacena Consulting, Natick, MA, USA

J. G. Still

Clinical Development Solutions, LLC, Raleigh, NC, USA

L. Vrishabhendra

Medpace Clinical Pharmacology, Cincinnati, OH, USA intravenous ascending doses of apitegromab of $10,20,30 \mathrm{mg} / \mathrm{kg}$ or placebo.

Results: Following single ascending doses, the pharmacokinetic parameters of apitegromab appeared to be similar across all dose groups, following a biphasic pattern of decline in the concentration-time curve. The mean apparent terminal $t_{1 / 2}$ after single intravenous doses of apitegromab ranged from 24 to 31 days across dose groups. Dose-related increases were observed in $C_{\max }$ following multiple ascending doses. Single and multiple apitegromab doses resulted in dose-dependent and sustained increases in serum latent myostatin, indicating robust target engagement. Apitegromab was safe and well tolerated, on the basis of the adverse event (AE) profile with no clinically meaningful changes in baseline vital signs, electrocardiograms, or clinical laboratory parameters and no anti-drug antibody formation.

Conclusion: These results support continued investigation of apitegromab for the treatment of patients with milder forms (type 2 and 3 ) of spinal muscular atrophy.

Keywords: Apitegromab; Human monoclonal antibody; Myostatin; Pharmacodynamics; Pharmacokinetics; Phase 1 study; Safety; Spinal muscular atrophy; SRK-015; Tolerability 


\section{Key Summary Points}

Apitegromab (SRK-015) is an investigational, fully human, monoclonal antibody that specifically binds to proforms of myostatin, which include promyostatin and latent myostatin, inhibiting myostatin activation. It is proposed as monotherapy or as an additive therapy to improve muscle function in spinal muscular atrophy.

Single and multiple ascending doses of apitegromab were safe and well tolerated at doses up to $30 \mathrm{mg} / \mathrm{kg}$ in healthy subjects.

Apitegromab demonstrated linear, doseproportional pharmacokinetics. Serum latent myostatin revealed dose-dependent pharmacodynamics.

The results of this study support the continued development of apitegromab for the treatment of spinal muscular atrophy.

\section{DIGITAL FEATURES}

This article is published with digital features, including a summary slide, to facilitate understanding of the article. To view digital features for this article go to https://doi.org/10.6084/ m9.figshare.14447298.

\section{INTRODUCTION}

Spinal muscular atrophy (SMA) is a debilitating neuromuscular disease caused by the loss of motor neuron function that can cause profound muscle atrophy and weakness [1]. Autosomal recessive proximal SMA, or 5q-SMA, is the most prevalent form, accounting for up to $95 \%$ of cases. This form is caused by deletions of the survival motor neuron-1 gene (SMN1) [1]. Two nearly identical SMN genes (SMN1 and SMN2) exist on chromosome 5q13. Both produce identical full-length SMN protein; however, a mutation in a splice acceptor site in SMN2 primarily results in truncated, unstable transcripts, with only about $10 \%$ producing full-length protein [2]. SMA results from the homozygous deletion of the SMN1 gene [3] and SMN2 expression of shortened, unstable, and rapidly degraded isoform of SMN [1]. The net effect of SMN1 deletion is diminished levels of fulllength, stable SMN protein. Without this protein, anterior horn cells degenerate, resulting in skeletal muscle atrophy and weakness [4]. Disease severity is determined by the number of copies of SMN2, with greater numbers associated with milder phenotypes [1].

Clinically, there are five types of SMA $[1,4]$. Type 0 is the most severe which has in utero onset with reduced or absent movements, requiring mechanical ventilation at birth. Infants with type 1 are very weak and unable to sit, children with type 2 are non-ambulant but able to sit upright, while type 3 children are ambulatory. Some type 3 patients can sit and walk unassisted but may lose that ability over time. Adult-onset, type 4 SMA, is the mildest form with minor muscle-weakness. Although these are traditional classifications, they are changing because of increased newborn screening and pre-symptomatic treatment [5].

SMN correctors, also known as SMN upregulators, have recently been approved for treating patients with SMA [6]. These therapies introduce an intact SMN1 gene or increase expression of full-length SMN protein from the related SMN2 gene [6]. Although SMN upregulators improve neuromotor tone across SMA types, patients still exhibit motor function deficits $[7,8]$. SMN upregulators may stabilize the disease course but cannot reverse the muscle atrophy that characterizes SMA [9].

Myostatin (growth and differentiation factor 8; GDF-8) is a negative regulator of skeletal muscle mass [10]. Humans and animals born with myostatin mutations develop a hypermuscular, but otherwise healthy phenotype [11-13]. Myostatin is initially produced in skeletal muscle as an inactive precursor associated with the extracellular matrix, termed promyostatin [10]. An initial proteolytic step 
processes promyostatin into a primed state, termed latent myostatin, which is primarily detected in serum [10]. A second cleavage event converts the latent myostatin protein into the mature growth factor which binds to its receptor and initiates a downstream cascade of events via the SMAD2/3 complex, leading to protein breakdown and muscle atrophy [14]. Inhibiting myostatin signalling may provide therapeutic benefit for patients with muscle atrophy or muscle-wasting disease.

Previous investigations assessing the use of myostatin antibodies to treat neuromuscular disorders $[15,16]$ and cancer-related cachexia [17] achieved limited success. There were no improvements in muscle strength or function in subjects with muscular dystrophy or elderly subjects with low muscle strength $[15,16]$ and no clinical benefit among patients with cancer [17].

In muscular dystrophy, muscle tissues are structurally damaged and may not benefit from added muscle mass. As active mature myostatin shares considerable homology with other TGF $\beta$ superfamily members and binds to the same receptor, the lack of myostatin specificity may result in cross-reaction with other TGF $\beta$ family members, raising safety concerns $[18,19]$.

In contrast, apitegromab (SRK-015) is an investigational, fully human, monoclonal antibody that specifically binds to proforms of myostatin, which include promyostatin and latent myostatin, inhibiting myostatin activation [10]. By targeting its precursors, apitegromab prevents release of the active mature myostatin and subsequent binding to its muscle surface receptor [10]. In vitro binding studies demonstrate that apitegromab does not bind the mature myostatin growth factor and does not bind to any form of GDF-11, activin A, or the mature forms of BMP9/10 or TGF $\beta 1$ which all share the same receptor [10]. Results from preclinical studies also demonstrate that promyostatin is the predominant form of myostatin in skeletal muscle, allowing apitegromab to inhibit myostatin activation directly in the target tissue $[10,20]$.

Using the SMN $\Delta 7$ mouse model of SMA, we previously demonstrated that post-symptomatic SMN restoration (beginning at postnatal day 24) in combination with muSRK015P, the parental clone of apitegromab, resulted in significant increases in muscle strength and function compared to mice treated with an SMN upregulator alone [21]. Similar results were observed in SMN $\Delta 7$ mice treated pre-symptomatically with muSRK-015P [21]. These studies also demonstrated the ability of apitegromab to engage latent myostatin, to an equal extent, across both late and early SMN restoration mouse models, despite significantly lower circulating latent myostatin levels in the more severe model of later SMN restoration. These data indicate that in mouse models of SMA, the muscle produces sufficient levels of myostatin for therapeutic inhibition to be effective, and that circulating latent myostatin may simply reflect overall muscle mass [21].

The objective of this phase 1 study was to investigate the safety of single and multiple doses of apitegromab across the planned therapeutic dose range to support future clinical studies. This was a randomized, double-blind, placebo-controlled, sequential cohort, two-part, single ascending dose (SAD) and multiple ascending dose (MAD) study of apitegromab in healthy adult subjects (Fig. 1). The purpose was to assess the safety, tolerability, pharmacokinetic (PK) parameters, and pharmacodynamic (PD) profile of apitegromab. The primary objective was to evaluate the safety and tolerability of single and multiple apitegromab doses up to $30 \mathrm{mg} / \mathrm{kg}$ by intravenous (IV) infusion. Secondary objectives were to evaluate the PK parameters of single and multiple doses of apitegromab up to $30 \mathrm{mg} / \mathrm{kg}$ by IV infusion and assess the immunogenicity of apitegromab.

\section{METHODS}

\section{Subject Eligibility}

Eligible participants were healthy male and female subjects, $18-55$ years old, weighing at least $60 \mathrm{~kg}$ (men) and at least $50 \mathrm{~kg}$ (women) with a BMI of $18.5-34.9 \mathrm{~kg} / \mathrm{m}^{2}$. Female subjects of childbearing potential agreed to use two medically accepted forms of contraception from the screening period through the final study 


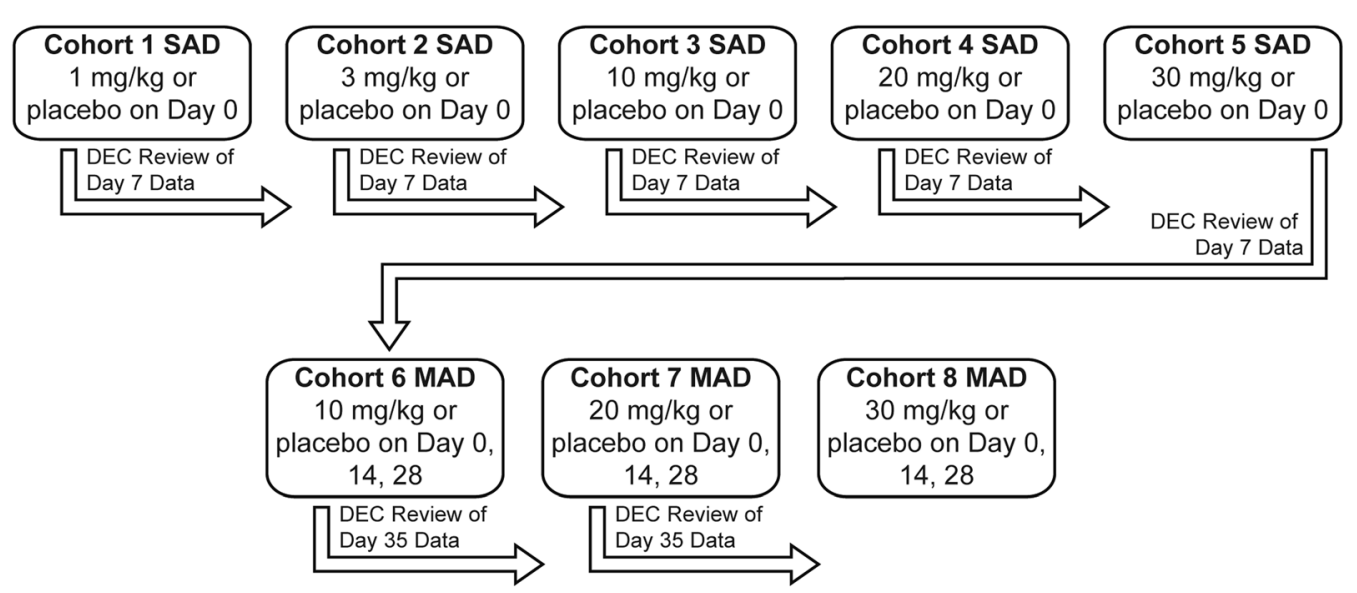

Fig. 1 Study design. In part A (single ascending dose, $S A D)$, an initial sentinel group of subjects in each cohort (one active, one placebo) were administered apitegromab to allow for initial safety assessments. A minimum of $24 \mathrm{~h}$ elapsed after the start of infusion of the last sentinel subject before the remainder of the cohort was treated. Dosing of subjects 3-8 proceeded if no safety issues were identified by the Dose Escalation Committee (DEC) for the first two

visit. Male subjects with female partners of childbearing potential agreed to use a highly effective, medically acceptable form of contraception from the screening period through the final study visit or at least 90 days after the last dose [47].

Reasons for exclusion from the study included acute or chronic illness at screening; history of exposure to a $\mathrm{mAb}$ or $\mathrm{Fc}$ domain-bearing recombinant protein within 5 years prior to screening and history of hypersensitivity reactions with exposure; exposure to a biologic within 90 days prior to screening or live vaccine within 14 days prior to screening; any current or prior use of anabolic steroids, systemic corticosteroids, systemic beta-adrenergic agonists; use of dietary or herbal supplements and vitamins, including protein supplements, growth hormones, or other substances designed to affect muscle growth [47]. Subjects were also excluded if they participated in strenuous exercise within 14 days prior to study day -1 , donated blood (more than $450 \mathrm{~mL}$ ) or serum within 60 days, or had a history of substance abuse or dependency. subjects in each cohort. In part B (multiple ascending dose, MAD), subjects received apitegromab or placebo every 2 weeks on days 0,14 , and 28 . Initiation of part B did not occur until the planned cumulative apitegromab dose for part B $(30 \mathrm{mg} / \mathrm{kg})$ had been administered as a single dose to the full cohort in part A and the DEC had determined that it was safe to proceed

\section{Study Design and Treatment}

This study was conducted in two parts. Part A was the SAD phase while part $B$ was the MAD phase. Eligibility screening for parts A and B was performed up to 28 days prior to day 0 dosing. Subjects were admitted to the phase 1 study unit the day prior to each infusion. No subjects enrolled into part A were later enrolled into part B, nor were part B subjects enrolled into part A.

Subject demographics, current and past medical status, history of surgery, allergies, and concomitant medications were recorded. Vital signs and a complete physical examination were performed by the investigator or a qualified designee within $120 \mathrm{~min}$ prior to each infusion.

\section{Part A: Single Ascending Dose, Dose Escalation}

The doses (Table 1; Fig. 1) for part A (SAD) were $1 \mathrm{mg} / \mathrm{kg}$ (cohort 1 ), $3 \mathrm{mg} / \mathrm{kg}$ (cohort 2 ), $10 \mathrm{mg} /$ $\mathrm{kg}$ (cohort 3), $20 \mathrm{mg} / \mathrm{kg}$ (cohort 4), and $30 \mathrm{mg} /$ $\mathrm{kg}$ (cohort 5). Eight subjects were enrolled in 
Table 1 Treatment assignment by study part and cohort

\begin{tabular}{lll}
\hline Study part & Cohort & Treatment assignment \\
\hline A (SAD) & 1 & $1 \mathrm{mg} / \mathrm{kg}$ apitegromab $(n=6)$ or placebo $(n=2)$ on day 0 \\
& 2 & $3 \mathrm{mg} / \mathrm{kg}$ apitegromab $(n=6)$ or placebo $(n=2)$ on day 0 \\
& 3 & $10 \mathrm{mg} / \mathrm{kg}$ apitegromab $(n=6)$ or placebo $(n=2)$ on day 0 \\
& 4 & $20 \mathrm{mg} / \mathrm{kg}$ apitegromab $(n=6)$ or placebo $(n=2)$ on day 0 \\
B (MAD) & 5 & $30 \mathrm{mg} / \mathrm{kg}$ apitegromab $(n=6)$ or placebo $(n=2)$ on day 0 \\
& 6 & $10 \mathrm{mg} / \mathrm{kg}$ apitegromab $(n=6)$ or placebo $(n=2)$ on days 0,14, and 28 \\
& 7 & $20 \mathrm{mg} / \mathrm{kg}$ apitegromab $(n=6)$ or placebo $(n=2)$ on days 0,14, and 28 \\
& 8 & $30 \mathrm{mg} / \mathrm{kg}$ apitegromab $(n=6)$ or placebo $(n=2)$ on days 0,14, and 28 \\
\hline
\end{tabular}

$S A D$ single ascending dose, $M A D$ multiple ascending dose

each cohort and randomized 3:1 to receive either active apitegromab $(n=6)$ or matching placebo $(n=2)$ administered as an IV infusion. In part $A$, an initial sentinel group of two subjects in each cohort (one active, one placebo) were infused to permit initial safety assessments. A minimum of $24 \mathrm{~h}$ elapsed after the start of infusion of the last sentinel subject before the remainder of the cohort was treated. Dosing of subjects 3-8 proceeded if no safety issues were identified by the Dose Escalation Committee (DEC) for the first two subjects in each cohort.

All subjects in part A had continuous cardiac monitoring for approximately $12 \mathrm{~h}$ after dosing and were observed as inpatients for 2 days $(48 \mathrm{~h})$ after dosing. Following discharge from the clinic, subjects returned to the phase 1 unit for follow-up visits on days 7, 14, 21, 28, 42, 56, 84, and 112 for safety monitoring and blood sampling for immunogenicity, PK, and PD assessments. Safety data from each cohort were reviewed by the DEC prior to escalation to the next higher dose.

\section{Part B: Multiple Ascending Dose, Dose escalation}

During part B (MAD) (Table 1; Fig. 1), three cohorts received apitegromab $10 \mathrm{mg} / \mathrm{kg}$ (cohort 6), $20 \mathrm{mg} / \mathrm{kg}$ (cohort 7), and $30 \mathrm{mg} / \mathrm{kg}$ (cohort 8). Eight subjects were enrolled in each cohort and were randomized to receive apitegromab $(n=6)$ or matching placebo $(n=2)$ once every 2 weeks for a total of three repeat doses on days 0,14 , and 28 . Initiation of part $B$ did not occur until the planned cumulative apitegromab dose for part B (three doses of $10 \mathrm{mg} / \mathrm{kg}$ ) had been administered as a single dose to the full cohort in part A $(30 \mathrm{mg} / \mathrm{kg}$ in advance of part $\mathrm{B}$, three doses of $10 \mathrm{mg} / \mathrm{kg}$ ); and the DEC had determined that it was safe to proceed on the basis of a review of safety data through day 7 for the $30-\mathrm{mg} / \mathrm{kg}$ SAD cohort; and available safety data from earlier SAD cohorts. All subjects in part B had continuous cardiac monitoring for approximately $12 \mathrm{~h}$ and were observed as inpatients for the first $48 \mathrm{~h}$ after each infusion. In addition to clinic admission for dosing on days 0,14 , and 28, subjects returned to the phase 1 unit for followup visits on days $7,21,35,42,56,84,112$, and 140 for safety monitoring and blood sampling for immunogenicity, PK and PD assessments.

\section{Pharmacokinetic and Pharmacodynamic Assessments}

Initial blood samples for PK and PD assessments were obtained within 15 min prior to apitegromab administration. For part A (SAD) and part B (MAD), blood samples for PK and serum latent myostatin (PD) were collected prior to infusion, at the end of infusion, and 4, 8, 24, and $48 \mathrm{~h}$ post-infusion. Additional samples were collected in part A on days 7, 14, 21, 28, 
$42,56,84$, and 112 and in part B on days 7,21 , $35,42,56,84,112$, and 140 .

PK samples were analyzed by a validated sensitive plate-based electrochemiluminescence (ECL) assay developed to quantitate apitegromab in human serum with a lower limit of quantification of $200 \mathrm{ng} / \mathrm{mL}$. Immunogenicity was assessed in all subjects who received apitegromab. Latent myostatin concentrations were measured with a qualified plate-based ECL immunoassay to quantitate latent myostatin in serum samples. The assay selectively recognizes latent myostatin and does not detect promyostatin, mature myostatin, or other closely related members of the TGF $\beta$ superfamily. The assay has a quantitation range of $2.3-5000 \mathrm{ng} / \mathrm{mL}$ and is tolerant up to $2.5 \mathrm{mg} / \mathrm{mL}$ of apitegromab.

Single-dose PK parameters were estimated using noncompartmental analysis (part A), while the multiple dose PK parameters were estimated using noncompartmental and compartmental methods (part B). Phoenix ${ }^{\circledR}$ WinNonlin ${ }^{\circledR}$ (Version 8.3; Princeton, NJ) was used to estimate these parameters. Only noncompartmental analysis results are presented in this publication.

\section{Safety Assessments}

\section{Anti-Drug Antibody Testing}

The presence of anti-drug antibodies was evaluated using a validated plate-based ECL bridging immunoassay. Both screening and confirmatory portions of the assay have a sensitivity of $100 \mathrm{ng} / \mathrm{mL}$.

\section{Laboratory Assessments}

Clinical chemistry, hematology, and urinalysis assessments were performed using standard methods. Blood samples for laboratory assessments were obtained at the phase 1 unit by the study investigator or a trained designated staff member.

\section{Safety Assessments}

Safety was monitored through reported adverse events (AEs), vital signs, physical examinations, clinical laboratory testing, and infusion site reactions. Subjects underwent continuous electrocardiogram (ECG) monitoring for approximately $12 \mathrm{~h}$ after each infusion. $\mathrm{AE}$ severity was graded using NCI CTCAE v5.0 [22].

\section{Statistical Analysis}

The sample size was chosen to provide adequate numbers of subjects to characterize the safety and tolerability of apitegromab. Safety analyses included randomized subjects who received at least one dose of apitegromab. PK assessments included subjects with at least one quantifiable apitegromab serum concentration. Data were described and analyzed using statistical software (SAS ${ }^{\circledR}$ System, v9.4; SAS Institute Inc., Cary, NC). Descriptive statistics included the number of subjects $(N)$, mean, standard deviation (SD), median, minimum, and maximum results.

\section{Ethics}

This study was approved by a commercial institutional review board (IRB; Advarra ${ }^{\circledR}$, Columbia, MD, \#00024966), complied with the ethical principles of Good Clinical Practice as required by the International Conference on Harmonisation, the Declaration of Helsinki as amended, and US Investigational New Drug regulations (21 CFR 56). In addition, the IRB approved the written informed consent form, including the ability to publish data from the study, any consent form updates, subject recruitment procedures (e.g., advertisements), and any written information provided to the subjects prior to implementation. All subjects provided informed written consent to participate in the study prior to any study-related activities.

\section{RESULTS}

\section{Study Population}

The study was conducted between 27 April 2018 and 11 March 2019, inclusive. Subject demographics and clinical characteristics are summarized in Tables 2 and 3. Sixty-four subjects were planned: 40 subjects (five cohorts of eight 
Table 2 Demographic and baseline characteristics, safety population: part A, single ascending dose

\begin{tabular}{|c|c|c|c|c|c|c|c|}
\hline Characteristic & $\begin{array}{l}\text { All } \\
\text { placebo } \\
(n=10)\end{array}$ & $\begin{array}{l}\text { Cohort } 1 \\
\text { Apitegromab } \\
1 \mathrm{mg} / \mathrm{kg} \\
(n=6)\end{array}$ & $\begin{array}{l}\text { Cohort } 2 \\
\text { Apitegromab } \\
3 \mathrm{mg} / \mathrm{kg} \\
(n=6)\end{array}$ & $\begin{array}{l}\text { Cohort } 3 \\
\text { Apitegromab } \\
10 \mathrm{mg} / \mathrm{kg} \\
(n=6)\end{array}$ & $\begin{array}{l}\text { Cohort } 4 \\
\text { Apitegromab } \\
20 \mathrm{mg} / \mathrm{kg} \\
(n=6)\end{array}$ & $\begin{array}{l}\text { Cohort } 5 \\
\text { Apitegromab } \\
30 \mathrm{mg} / \mathrm{kg} \\
(n=6)\end{array}$ & $\begin{array}{l}\text { All } \\
\text { apitegromab } \\
(n=30)\end{array}$ \\
\hline \multicolumn{8}{|c|}{ Age at informed consent (years) } \\
\hline Mean (SD) & $\begin{array}{l}39.1 \\
\quad(12.9)\end{array}$ & $33.7(14.0)$ & $28.7(1.5)$ & $32.3(7.8)$ & $36.5(12.8)$ & $43.8(9.0)$ & $35.0(10.7)$ \\
\hline $\begin{array}{l}\text { Median (min, } \\
\max )\end{array}$ & $\begin{array}{c}36.0(20, \\
55)\end{array}$ & $30.0(20,53)$ & $28.0(27,31)$ & $34.5(21,42)$ & $32.5(22,52)$ & $48.5(30,51)$ & $32.5(20,53)$ \\
\hline \multicolumn{8}{|l|}{ Gender, $n(\%)$} \\
\hline Female & $4(40.0)$ & $4(66.7)$ & $1(16.7)$ & $2(33.3)$ & $1(16.7)$ & $3(50.0)$ & $11(36.7)$ \\
\hline Male & $6(60.0)$ & $2(33.3)$ & $5(83.3)$ & $4(66.7)$ & $5(83.3)$ & $3(50.0)$ & $19(63.3)$ \\
\hline \multicolumn{8}{|l|}{ Race, $n(\%)$} \\
\hline White & $4(40.0)$ & $0(0)$ & $0(0)$ & $1(16.7)$ & $3(50.0)$ & $1(16.7)$ & $5(16.7)$ \\
\hline $\begin{array}{l}\text { Black/African } \\
\text { American }\end{array}$ & $5(50.0)$ & $6(100)$ & $6(100)$ & $5(83.3)$ & $3(50.0)$ & $5(83.3)$ & $25(83.3)$ \\
\hline $\begin{array}{l}\text { American } \\
\text { Indian/ } \\
\text { Alaska } \\
\text { Native }\end{array}$ & $1(10.0)$ & $0(0)$ & $0(0)$ & $0(0)$ & $0(0)$ & $0(0)$ & $0(0)$ \\
\hline \multicolumn{8}{|l|}{ Ethnicity, $n$ (\%) } \\
\hline $\begin{array}{l}\text { Hispanic or } \\
\text { Latino }\end{array}$ & $1(10.0)$ & $0(0)$ & $1(16.7)$ & $0(0)$ & $0(0)$ & $0(0)$ & $1(3.3)$ \\
\hline $\begin{array}{l}\text { Not Hispanic } \\
\text { or Latino }\end{array}$ & $9(90.0)$ & $6(100)$ & $5(83.3)$ & $4(66.7)$ & $6(100)$ & $6(100)$ & $27(90)$ \\
\hline Not reported & $0(0)$ & $0(0)$ & $0(0)$ & $2(33.3)$ & $0(0)$ & $0(0)$ & $2(6.7)$ \\
\hline \multicolumn{8}{|c|}{ Baseline body weight (kg) } \\
\hline Mean (SD) & $\begin{array}{l}78.0 \\
(10.5)\end{array}$ & $76.9(8.1)$ & $88.5(6.5)$ & $86.4(10.7)$ & $75.6(16.4)$ & $80.8(11.9)$ & $81.6(11.7)$ \\
\hline $\begin{array}{l}\text { Median (min, } \\
\max )\end{array}$ & $\begin{array}{c}78.6 \\
(63.8 \\
95.3)\end{array}$ & $\begin{array}{c}80.5(66.1 \\
85.5)\end{array}$ & $\begin{array}{c}89.4(79.8 \\
98.8)\end{array}$ & $\begin{array}{c}82.7(74.7 \\
102.2)\end{array}$ & $\begin{array}{c}72.5(57.8 \\
104.2)\end{array}$ & $\begin{array}{c}78.3(68.5 \\
102.7)\end{array}$ & $\begin{array}{c}80.9(57.8 \\
104.2)\end{array}$ \\
\hline \multicolumn{8}{|c|}{ Baseline BMI $\left(\mathrm{kg} / \mathrm{m}^{2}\right)$} \\
\hline Mean (SD) & $\begin{array}{l}27.1 \\
(4.7)\end{array}$ & $27.0(3.1)$ & $30.2(3.1)$ & $28.3(2.3)$ & $25.9(4.1)$ & $27.9(3.2)$ & $27.9(3.3)$ \\
\hline
\end{tabular}


Table 2 continued

\begin{tabular}{|c|c|c|c|c|c|c|c|}
\hline Characteristic & $\begin{array}{l}\text { All } \\
\text { placebo } \\
(n=10)\end{array}$ & $\begin{array}{l}\text { Cohort } 1 \\
\text { Apitegromab } \\
1 \mathrm{mg} / \mathrm{kg} \\
(n=6)\end{array}$ & $\begin{array}{l}\text { Cohort } 2 \\
\text { Apitegromab } \\
3 \mathrm{mg} / \mathrm{kg} \\
(n=6)\end{array}$ & $\begin{array}{l}\text { Cohort } 3 \\
\text { Apitegromab } \\
10 \mathrm{mg} / \mathrm{kg} \\
(n=6)\end{array}$ & $\begin{array}{l}\text { Cohort } 4 \\
\text { Apitegromab } \\
20 \mathrm{mg} / \mathrm{kg} \\
(n=6)\end{array}$ & $\begin{array}{l}\text { Cohort } 5 \\
\text { Apitegromab } \\
30 \mathrm{mg} / \mathrm{kg} \\
(n=6)\end{array}$ & $\begin{array}{l}\text { All } \\
\text { apitegromab } \\
(n=30)\end{array}$ \\
\hline $\begin{array}{l}\text { Median } \\
(\min , \max )\end{array}$ & $\begin{array}{l}26.1 \\
\quad(20.3, \\
33.8)\end{array}$ & $\begin{array}{c}26.7(22.8 \\
31.8)\end{array}$ & $\begin{array}{c}30.0(26.1 \\
34.4)\end{array}$ & $\begin{array}{c}28.9(25.1 \\
30.9)\end{array}$ & $\begin{array}{c}24.3(22.1 \\
33.1)\end{array}$ & $\begin{array}{c}27.0(23.5 \\
32.9)\end{array}$ & $\begin{array}{c}27.9(22.1 \\
34.4)\end{array}$ \\
\hline
\end{tabular}

Baseline is defined as data collected on day -1 ; if the baseline measurements on day -1 are missing, the last measurement prior to dosing served as baseline. Subjects receiving placebo are pooled together for part A (single ascending dose). Percentages are calculated using the number of subjects in the safety population in each column as the denominator $B M I$ body mass index, max maximum, min minimum

subjects) in part A (SAD) and 24 subjects (three cohorts, eight subjects each) in part B (MAD). The safety population in both parts of the study included 66 randomized subjects.

In part A (Table 4), subjects were randomized to apitegromab $(n=30)$ or placebo $(n=10)$ and included in the safety population. The study was completed by all placebo-treated subjects and most $(n=29)$ apitegromab-treated subjects. One subject in the apitegromab group was lost to follow-up.

In part B (Table 5), subjects were randomized to apitegromab $(n=20)$ or placebo $(n=6)$ and included in the safety population. The study was completed by four placebo-treated subjects and 15 apitegromab-treated subjects. As two subjects in cohort 6 withdrew from the study prior to receiving the second dose, two additional subjects were enrolled to maintain the required number of subjects.

\section{Tolerability and Safety}

In part A, AEs occurred in subjects treated with placebo $(n=4)$ and apitegromab $(n=7)$. Most were of mild severity and none led to discontinuation. In part $B$, mild AEs occurred in placebo subjects $(n=4)$ while moderate AEs were reported in apitegromab subjects $(n=4)$ and included ligament sprain, viral infection, tooth abscess, and upper respiratory tract infection. Drug-related AEs of postural dizziness occurred in subjects receiving placebo $(n=1)$ and apitegromab $(n=1)$. No clinically significant changes occurred in the clinical chemistry, hematology, coagulation, and urinalysis parameters and no clinically meaningful trends in baseline vital signs or ECGs.

A 55-year-old man enrolled in part B was randomized to apitegromab $10 \mathrm{mg} / \mathrm{kg}$. On study day 88 , he presented to the emergency department with a 2-h history of radiating abdominal pain, diaphoresis, restlessness, nausea, and recent emesis. Computed tomography confirmed gallstone-induced pancreatitis and pancreatic edema. The subject was withdrawn from the study and eventually recovered. The event was not drug-related. AEs reported in parts A and $B$ are summarized in Tables 6 and 7.

\section{Pharmacokinetics}

\section{Single Ascending Dose Pharmacokinetics}

After IV administration of apitegromab $1-30 \mathrm{mg} / \mathrm{kg}$ in the part A (SAD) group, quantifiable concentrations of serum apitegromab were measured after the end of the infusion (Table 8; Fig. 2). After single doses of apitegromab were infused over $120 \mathrm{~min}$, a biphasic pattern of decline was apparent in the concentration-time curves (Fig. 2); however, the number of compartments may depend on the limits of detection. The apitegromab $C_{\max }$ occurred soon after completing the IV infusion. Mean serum concentrations, $C_{\max }$, and AUC values increased proportionally with increasing 
Table 3 Demographic and baseline characteristics, safety population: part B, multiple ascending dose

\begin{tabular}{|c|c|c|c|c|c|}
\hline Characteristic & $\begin{array}{l}\text { All placebo } \\
(n=6)\end{array}$ & $\begin{array}{l}\text { Cohort } 6 \\
\text { Apitegromab } \\
10 \mathrm{mg} / \mathrm{kg} \\
(n=8)\end{array}$ & $\begin{array}{l}\text { Cohort } 7 \\
\text { Apitegromab } \\
20 \mathrm{mg} / \mathrm{kg} \\
(n=6)\end{array}$ & $\begin{array}{l}\text { Cohort } 8 \\
\text { Apitegromab } \\
30 \mathrm{mg} / \mathrm{kg} \\
(n=6)\end{array}$ & $\begin{array}{l}\text { All apitegromab } \\
(n=20)\end{array}$ \\
\hline \multicolumn{6}{|c|}{ Age at informed consent (years) } \\
\hline Mean (SD) & $43.2(8.5)$ & $36.4(8.9)$ & $39.3(10.8)$ & $35.0(13.1)$ & $36.9(10.4)$ \\
\hline Median $(\min , \max )$ & $41.0(34,54)$ & $34.5(27,54)$ & $41.0(26,52)$ & $36.0(18,50)$ & $36.5(18,54)$ \\
\hline \multicolumn{6}{|l|}{ Gender, $n(\%)$} \\
\hline Female & $3(50.0)$ & $5(62.5)$ & $2(33.3)$ & $3(50.0)$ & $10(50.0)$ \\
\hline Male & $3(50.0)$ & $3(37.5)$ & $4(66.7)$ & $3(50.0)$ & $10(50.0)$ \\
\hline \multicolumn{6}{|l|}{ Race, $n(\%)$} \\
\hline White & $4(66.7)$ & $2(25.0)$ & $3(50.0)$ & $1(16.7)$ & $6(30.0)$ \\
\hline $\begin{array}{l}\text { Black/African } \\
\text { American }\end{array}$ & $2(33.3)$ & $5(62.5)$ & $3(50.0)$ & $4(66.7)$ & $12(60.0)$ \\
\hline Other & $0(0)$ & $1(12.5)$ & $0(0)$ & $1(16.7)$ & $2(10)$ \\
\hline \multicolumn{6}{|l|}{ Ethnicity, $n(\%)$} \\
\hline Hispanic/Latino & $0(0)$ & $2(25.0)$ & $0(0)$ & $0(0)$ & $2(10.0)$ \\
\hline Not Hispanic/Latino & $6(100)$ & $6(75.0)$ & $6(100)$ & $6(100)$ & $18(90.0)$ \\
\hline \multicolumn{6}{|l|}{ Baseline body weight (kg) } \\
\hline Mean (SD) & $83.6(11.7)$ & $83.7(16.0)$ & $79.6(10.5)$ & $84.0(16.6)$ & $82.5(14.2)$ \\
\hline Median (min, $\max )$ & $86.6(62.2,97.3)$ & $84.4(59.2,107.2)$ & $80.1(66.6,90.5)$ & $89.0(52.2,100.5)$ & $87.1(52.2,107.2)$ \\
\hline \multicolumn{6}{|l|}{ Baseline BMI $\left(\mathrm{kg} / \mathrm{m}^{2}\right)$} \\
\hline Mean (SD) & $27.1(3.2)$ & $30.2(3.3)$ & $26.7(3.4)$ & $28.0(4.2)$ & $28.5(3.8)$ \\
\hline Median $(\min , \max )$ & $26.8(22.5,31.4)$ & $30.8(24.1,33.4)$ & $27.7(21.5,29.6)$ & $28.7(20.1,32.6)$ & $29.2(20.1,33.4)$ \\
\hline
\end{tabular}

Baseline is defined as data collected on day -1 ; if the baseline measurements on day -1 are missing, the last measurement prior to dosing served as baseline. Subjects receiving placebo were pooled together for part B (multiple ascending dose). Percentages were calculated using the number of subjects in the safety population in each column as the denominator $B M I$ body mass index, max maximum, min minimum

dose (Table 8; Fig. 2). The drug clearance (CL) of apitegromab was low $(6.05-7.69 \mathrm{~mL} / \mathrm{h})$ and not dose-dependent. The mean apparent terminal $t_{1 / 2}$ ranged from 580 to $739 \mathrm{~h}$ (24-31 days) across the five dose groups. The mean volume of distribution $\left(V_{\mathrm{z}}\right)$ of apitegromab was dose-independent and ranged from 5.40 to $6.85 \mathrm{~L}$, indicating that the drug resided in both vascular and extravascular spaces.

\section{Multiple Ascending Dose Pharmacokinetics}

Following the administration of multiple ascending apitegromab doses of $10-30 \mathrm{mg} / \mathrm{kg}$, quantifiable serum concentrations of apitegromab were detected at the end of infusion after every dose and prior to the administration of the subsequent doses. Multiple-dose mean PK values by dose group after 140 days are shown in Table 9. Dose-related increases were observed 


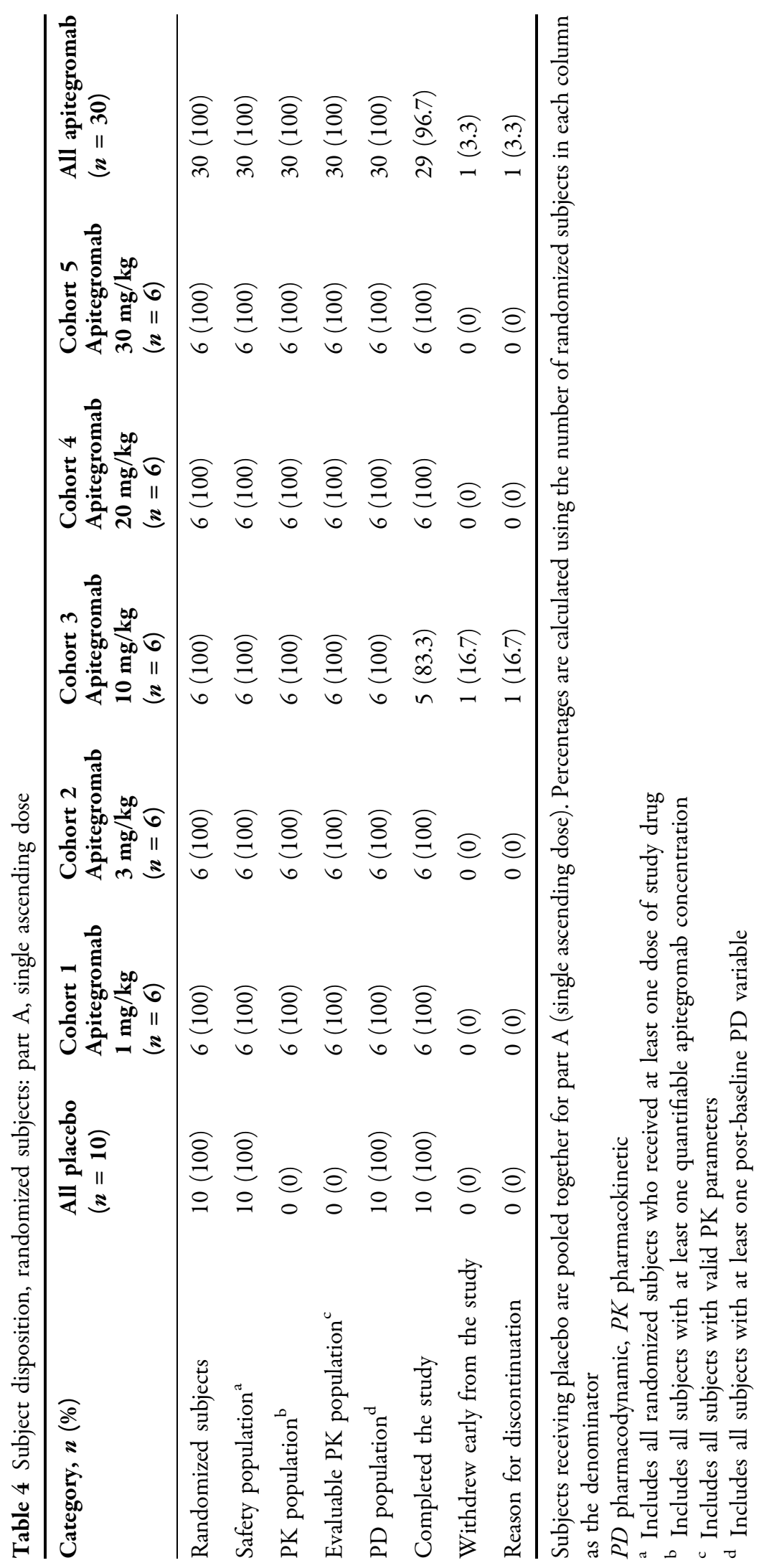


Table 5 Subject disposition, randomized subjects: part B, multiple ascending doses

\begin{tabular}{|c|c|c|c|c|c|}
\hline Category, $n(\%)$ & $\begin{array}{l}\text { All placebo } \\
(n=6)\end{array}$ & $\begin{array}{l}\text { Cohort } 6 \\
\text { Apitegromab } \\
10 \mathrm{mg} / \mathrm{kg} \\
(n=8)\end{array}$ & $\begin{array}{l}\text { Cohort } 7 \\
\text { Apitegromab } \\
20 \mathrm{mg} / \mathrm{kg} \\
(n=6)\end{array}$ & $\begin{array}{l}\text { Cohort } 8 \\
\text { Apitegromab } \\
30 \mathrm{mg} / \mathrm{kg} \\
(n=6)\end{array}$ & $\begin{array}{l}\text { All apitegromab } \\
(n=20)\end{array}$ \\
\hline Randomized subjects & $6(100)$ & $8(100)$ & $6(100)$ & $6(100)$ & $20(100)$ \\
\hline Safety population ${ }^{\mathrm{a}}$ & $6(100)$ & $8(100)$ & $6(100)$ & $6(100)$ & $20(100)$ \\
\hline PK population ${ }^{\mathrm{b}}$ & $0(0)$ & $8(100)$ & $6(100)$ & $6(100)$ & $20(100)$ \\
\hline Evaluable PK population $^{c}$ & $0(0)$ & $8(100)$ & $6(100)$ & $6(100)$ & $20(100)$ \\
\hline PD population ${ }^{\mathrm{d}}$ & $6(100)$ & $8(100)$ & $6(100)$ & $6(100)$ & $20(100)$ \\
\hline Completed the study & $4(66.7)$ & $4(50.0)$ & $6(100)$ & $5(83.3)$ & $15(75.0)$ \\
\hline Withdrew early from the study & $2(33.3)$ & $4(50.0)$ & $0(0)$ & $1(16.7)$ & $5(25.0)$ \\
\hline \multicolumn{6}{|l|}{ Primary reason for discontinuation } \\
\hline Lost to follow-up & $0(0)$ & $1(12.5)$ & $0(0)$ & $0(0)$ & $1(5.0)$ \\
\hline Sponsor or investigator decision & $0(0)$ & $1(12.5)$ & $0(0)$ & $0(0)$ & $1(5.0)$ \\
\hline Withdrawal by subject & $2(33.3)$ & $2(25.0)$ & $0(0)$ & $1(16.7)$ & $3(15.0)$ \\
\hline
\end{tabular}

Subjects receiving placebo are pooled together for part B (multiple ascending dose). Percentages are calculated using the number of randomized subjects in each column as the denominator

$P D$ pharmacodynamics, $P K$ pharmacokinetic

${ }^{a}$ Includes all randomized subjects who received at least one dose of study drug

b Includes all subjects with at least one quantifiable apitegromab concentration

c Includes all subjects with valid PK parameters

${ }^{\mathrm{d}}$ Includes all subjects with at least one postbaseline PD variable

in apitegromab $C_{\text {last }}$ and $C_{\text {max }}$. Estimates of $\mathrm{CL}$, $V_{\mathrm{z}}$, and $t_{1 / 2}$ using noncompartmental PK analyses were obtained using all data available for 18 patients who received active treatment (Table 9).

The overall mean CL ranged from 8.19 to $10.3 \mathrm{~mL} / \mathrm{h}$ and overall mean volume of distribution $\left(V_{\mathrm{z}}\right)$ ranged from 7.22 to $8.69 \mathrm{~L}$ for multiple dosing, after the last dose. These were within the range of values reported after single dosing, indicating stable PK over time. There was an accumulation of apitegromab from administration of the first dose to the last dose, with the mean accumulation ratio based on maximum concentrations (repeated $C_{\max }$ ) values of 1.42, 2.11, and 2.11 in the 10-, 20-, and $30-\mathrm{mg} / \mathrm{kg}$ dose cohorts, respectively. Mean $\mathrm{AUC}_{(0-336)}$ accumulation ratios of $1.9,2.6$, and 2.9 were observed in the 10-, 20-, and $30-\mathrm{mg} / \mathrm{kg}$ dose cohorts, respectively. The accumulation was expected owing to the observed range in median $t_{1 / 2}$ in part A of the study (580-739 h, independent of dose).

\section{Single Ascending Dose Pharmacodynamics}

After apitegromab administration, mean serum latent myostatin concentrations increased and plateaued between days 14 and 28, for doses up to $10 \mathrm{mg} / \mathrm{kg}$, and between days 42 and 56 for the 20 and $30 \mathrm{mg} / \mathrm{kg}$ doses, after which a decline in latent myostatin concentrations occurred in all dose cohorts (Fig. 2). Mean serum latent myostatin concentrations remained at or near baseline levels after placebo administration until day 112 (end of study). Overall, the change in latent myostatin levels for 20 and $30 \mathrm{mg} / \mathrm{kg}$ doses were not substantially different than the 3 and $10 \mathrm{mg} / \mathrm{kg}$ doses, though the duration of target engagement did trend with the dose. 
Table 6 Treatment-emergent adverse events (TEAEs), cohorts 1-5

\begin{tabular}{|c|c|c|c|c|c|c|}
\hline \multirow{2}{*}{ System organ class/preferred term, $n(\%)^{a}$} & \multirow{2}{*}{$\begin{array}{l}\text { Placebo } \\
(n=10)\end{array}$} & \multicolumn{5}{|l|}{ SRK-015 } \\
\hline & & $\begin{array}{l}1 \mathrm{mg} / \mathrm{kg} \\
(n=6)\end{array}$ & $\begin{array}{l}3 \mathrm{mg} / \mathrm{kg} \\
(n=6)\end{array}$ & $\begin{array}{l}10 \mathrm{mg} / \mathrm{kg} \\
(n=6)\end{array}$ & $\begin{array}{l}20 \mathrm{mg} / \mathrm{kg} \\
(n=6)\end{array}$ & $\begin{array}{l}30 \mathrm{mg} / \mathrm{kg} \\
(n=6)\end{array}$ \\
\hline Subjects with any TEAE & $5(50.0)$ & $3(50.0)$ & $3(50.0)$ & 0 & $2(33.3)$ & $1(16.7)$ \\
\hline Gastrointestinal disorders & $1(10.0)$ & 0 & 0 & 0 & 0 & $1(16.7)$ \\
\hline Gastroesophageal reflux disease & $1(10.0)$ & 0 & 0 & 0 & 0 & 0 \\
\hline Inguinal disease & 0 & 0 & 0 & 0 & 0 & $1(16.7)$ \\
\hline Infections and infestations & $1(10.0)$ & 0 & 0 & 0 & $2(33.3)$ & 0 \\
\hline Acute sinusitis & 0 & 0 & 0 & 0 & $1(16.7)$ & 0 \\
\hline Respiratory tract infection & 0 & 0 & 0 & 0 & $1(16.7)$ & 0 \\
\hline Sinusitis & $1(10.0)$ & 0 & 0 & 0 & 0 & 0 \\
\hline Injury, poisoning, and procedural complications/ & $2(20.0)$ & 0 & $2(33.3)$ & 0 & 0 & 0 \\
\hline Ligament sprain & $1(10.0)$ & 0 & $1(16.7)$ & 0 & 0 & 0 \\
\hline Contusion & $1(10.0)$ & 0 & 0 & 0 & 0 & 0 \\
\hline Laceration & 0 & 0 & $1(16.7)$ & 0 & 0 & 0 \\
\hline Nervous system disorders & 0 & $3(50.0)$ & $1(16.7)$ & 0 & 0 & 0 \\
\hline Headache & 0 & $3(50.0)$ & 0 & 0 & & 0 \\
\hline Migraine & 0 & 0 & $1(16.7)$ & 0 & 0 & 0 \\
\hline Respiratory, thoracic, and mediastinal disorders & 0 & $1(16.7)$ & 0 & 0 & 0 & 0 \\
\hline Rhinitis allergic & 0 & $1(16.7)$ & 0 & 0 & 0 & 0 \\
\hline Skin and subcutaneous tissue disorders & $1(10.0)$ & 0 & 0 & 0 & $1(16.7)$ & 0 \\
\hline Dermatitis contact & $1(10.0)$ & 0 & 0 & 0 & $1(16.7)$ & 0 \\
\hline Vascular disorders & 0 & $1(16.7)$ & 0 & 0 & 0 & 0 \\
\hline Hot flush & 0 & $1(16.7)$ & 0 & 0 & 0 & 0 \\
\hline
\end{tabular}

${ }^{a}$ MedDRA Medical Dictionary for Regulatory Activities

Multiple Ascending Dose Pharmacodynamics Mean serum latent myostatin concentrations increased after the first dose of apitegromab and remained elevated through the administration of the third dose (Fig. 2). Latent myostatin concentrations began to decline by day 56 in the 10 and $20 \mathrm{mg} / \mathrm{kg}$ dose cohorts, and day 84 in the $30 \mathrm{mg} / \mathrm{kg}$ dose cohorts. Mean serum latent myostatin concentrations remained at or near baseline levels after the administration of placebo until day 140 (end of study). Overall, apitegromab demonstrated pharmacodynamic target engagement and durable target saturation.

\section{Safety Signals}

No anti-drug antibodies (ADAs) were detected in subjects in part $\mathrm{A}(\mathrm{SAD})$ or part $\mathrm{B}$ (MAD) of the study. A single placebo subject in the apitegromab $10 \mathrm{mg} / \mathrm{kg}$ group was positive for 
Table 7 Treatment-emergent adverse events, cohorts 6-8

\begin{tabular}{|c|c|c|c|c|}
\hline \multirow[t]{2}{*}{ System organ class/preferred term, $n(\%)^{\mathrm{a}}$} & \multirow{2}{*}{$\begin{array}{l}\text { Placebo } \\
(n=6)\end{array}$} & \multicolumn{3}{|l|}{ SRK-015 } \\
\hline & & $\begin{array}{l}10 \mathrm{mg} / \mathrm{kg} \\
(n=8)\end{array}$ & $\begin{array}{l}20 \mathrm{mg} / \mathrm{kg} \\
(n=6)\end{array}$ & $\begin{array}{l}30 \mathrm{mg} / \mathrm{kg} \\
(n=6)\end{array}$ \\
\hline Subjects with any TEAE & $4(66.7)$ & $3(37.5)$ & $3(50.0)$ & $1(16.7)$ \\
\hline Gastrointestinal disorders & 0 & $1(12.5)$ & 0 & 0 \\
\hline Obstructive pancreatitis & 0 & $1(12.5)$ & 0 & 0 \\
\hline Infections and infestations & 0 & $3(37.5)$ & $3(50)$ & 0 \\
\hline Gastroenteritis & 0 & $1(12.5)$ & 0 & 0 \\
\hline Rhinitis & 0 & $1(12.5)$ & 0 & 0 \\
\hline Tooth abscess & 0 & 0 & $1(16.7)$ & 0 \\
\hline Upper respiratory tract infection & 0 & 0 & $1(16.7)$ & 0 \\
\hline Urinary tract infection & 0 & $1(12.5)$ & 0 & 0 \\
\hline Viral infection & 0 & 0 & $1(16.7)$ & 0 \\
\hline Viral upper respiratory tract infection & 0 & 0 & $1(16.7)$ & 0 \\
\hline Injury, poisoning, and procedural complications & $1(16.7)$ & $2(25.0)$ & $1(16.7)$ & 0 \\
\hline Muscle strain & 0 & $2(25.0)$ & 0 & 0 \\
\hline Ear abrasion & $1(16.7)$ & 0 & 0 & 0 \\
\hline Ligament sprain & 0 & 0 & $1(16.7)$ & 0 \\
\hline Musculoskeletal and connective tissue disorders & $1(16.7)$ & 0 & 0 & $1(16.7)$ \\
\hline Muscle spasms & 0 & 0 & 0 & $1(16.7)$ \\
\hline Musculoskeletal stiffness & $1(16.7)$ & 0 & 0 & 0 \\
\hline Nervous system disorders & $3(50.0)$ & 0 & $1(16.7)$ & 0 \\
\hline Dizziness postural & $2(33.3)$ & 0 & $1(16.7)$ & 0 \\
\hline Headache & $1(16.7)$ & 0 & 0 & 0 \\
\hline
\end{tabular}

${ }^{a} \operatorname{MedDRA}$ Medical Dictionary for Regulatory Activities

ADAs at baseline, but negative at every other time point tested. No subjects were positive for ADAs at any other visits in part B (MAD) of the study.

\section{DISCUSSION}

Apitegromab is a promyostatin and latent myostatin inhibitor monoclonal antibody under development to improve motor function in patients with SMA. Unlike other myostatin inhibitors, apitegromab selectively binds to promyostatin and latent myostatin with high affinity and inhibits function by occluding access to activating proteases. This selectivity prevents the release of mature myostatin, without inhibiting the function of other related growth factors, thereby avoiding any potential adverse reactions associated with non-selective anti-myostatin agents [10]. Safety signals reported among the less selective myostatin 
Table 8 Summary of pharmacokinetics parameters for apitegromab by treatment: part A, single ascending dose

\begin{tabular}{|c|c|c|c|c|c|}
\hline Noncompartmental PK parameter & $\begin{array}{l}\text { Cohort } 1 \\
1 \mathrm{mg} / \mathrm{kg} \\
(n=6)\end{array}$ & $\begin{array}{l}\text { Cohort } 2 \\
3 \mathrm{mg} / \mathrm{kg} \\
(n=6)\end{array}$ & $\begin{array}{l}\text { Cohort } 3 \\
10 \mathrm{mg} / \mathrm{kg} \\
(n=6)\end{array}$ & $\begin{array}{l}\text { Cohort } 4 \\
20 \mathrm{mg} / \mathrm{kg} \\
(n=6)\end{array}$ & $\begin{array}{l}\text { Cohort } 5 \\
30 \mathrm{mg} / \mathrm{kg} \\
(n=6)\end{array}$ \\
\hline Mean $C_{\max }, \mu \mathrm{g} / \mathrm{mL}(\mathrm{CV} \%)$ & $25.07(19.5)$ & $82.95(8.7)$ & $277.50(7.7)$ & $555.00(16.9)$ & $744.00(13.2)$ \\
\hline $\begin{array}{l}\text { Dose normalized } C_{\max },[\mu \mathrm{g} / \mathrm{mL}] / \\
{[\mathrm{mg} / \mathrm{kg}]}\end{array}$ & 25.07 & 27.65 & 27.75 & 27.75 & 24.8 \\
\hline Median $t_{1 / 2}, \mathrm{~h}(\min , \max )$ & $\begin{array}{l}738.8(617 \\
1078)\end{array}$ & $\begin{array}{l}599.1(533 \\
758)\end{array}$ & $\begin{array}{l}580.4(273 \\
702)\end{array}$ & $\begin{array}{l}585.3(384 \\
726)\end{array}$ & $\begin{array}{l}622.9(499 \\
723)\end{array}$ \\
\hline Mean CL, mL/h (CV\%) & $6.049(10.7)$ & $7.688(17.9)$ & $7.299(26.7)$ & $7.062(30.0)$ & $6.628(14.1)$ \\
\hline Mean $V_{\mathrm{z}}, \mathrm{L}(\mathrm{CV} \%)$ & $6.804(20.1)$ & $6.852(13.7)$ & $5.395(18.8)$ & $5.688(10.4)$ & $5.919(16.1)$ \\
\hline
\end{tabular}

Cohort $1(1 \mathrm{mg} / \mathrm{kg})$ anchored start times on the infusion when all other cohorts are based on post end of infusion. One subject in the $10 \mathrm{mg} / \mathrm{kg}$ group discontinued the study prior to day 14

$C L$ total body clearance, $C_{\max }$ maximum serum drug concentration, $C V$ coefficient of variation, max maximum, min minimum, $P K$ pharmacokinetics, $t_{1 / 2}$ terminal elimination half-life, $V_{z}$ volume of distribution

inhibitors including immunogenicity, ADAs, gingival bleeding, epistaxis, telangiectasias, and effects on the neurohormonal axes were not observed in this study [18, 19, 23, 24].

The binding affinity of apitegromab for promyostatin in $2.9 \mathrm{nM}$. The binding affinity for latent myostatin is $2.4 \mathrm{nM}$. No binding affinity, detected at $200 \mathrm{nM}$, was noted for myostatin, nor any other TGF $\beta$ family member. Activation of myostatin requires two distinct proteolysis events that generate the active mature growth factor. One proteolysis event occurs between promyostatin, predominantly found within the skeletal muscle, and latent myostatin, predominantly found in the serum, by the proprotein convertase. The other proteolytic event occurs between latent myostatin and the mature, active myostatin, via the tolloid protease. Apitegromab binds to both promyostatin and latent myostatin and inhibits tolloid-mediated cleavage of latent myostatin. The latent myostatin increases in serum were likely due to binding of apitegromab to its target in the muscle, resulting in transport of the antibody-antigen complex into the systemic circulation, until eventual clearance.

Previous myostatin inhibitor investigations focused on the treatment of facioscapulohumeral muscular dystrophy and Duchenne muscular dystrophy, with limited measurable benefit, likely because of structural tissue damage [23]. Apitegromab is being pursued in SMA because SMA is a condition primarily caused by impaired neuromotor innervation in otherwise structurally or mechanically sound muscle tissue.

Measurement of serum apitegromab levels demonstrated it achieved a linear, robust PK profile across the study, with generally low variability (less than 30\% CV) within each cohort. In part A (SAD), proportionality of apitegromab exposure was achieved at all dose levels $(1-30 \mathrm{mg} / \mathrm{kg})$ by comparing dose normalized $C_{\max }$ (Table 6). In part B (MAD), dose proportionality was achieved within each dosing period at the three dosing levels tested (Table 7), as assessed by $C_{\max }, C_{\text {last}}$, and AUC $_{(0-336)}$. Multiple apitegromab doses resulted in accumulating exposure as measured by comparing both the $C_{\max }$ after each dose and the $C_{\text {last }}$ within the first and second dosing interval (Table 7). In addition, the $\mathrm{AUC}_{(0-336)}$ showed similar trends toward accumulation over the three dosing periods. While steadystate apitegromab exposure could not be assessed after only three doses, the overall exposure trends indicate that steady state would be achieved after subsequent doses. The half-life, calculated from data in part A (SAD), ranged from 24 to 31 days. This long half-life suggests 


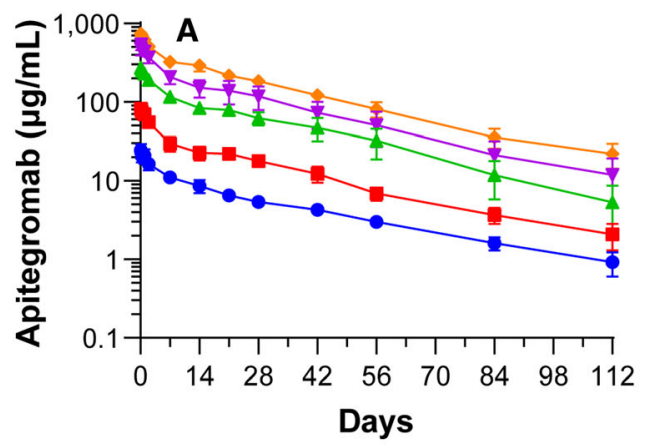

$\rightarrow$ Cohort $1,1 \mathrm{mg} / \mathrm{kg}(\mathrm{n}=6) \rightarrow$ Cohort $2,3 \mathrm{mg} / \mathrm{kg}(\mathrm{n}=6)$
- Cohort $3,10 \mathrm{mg} / \mathrm{kg}(\mathrm{n}=6) \rightarrow$ Cohort $4,20 \mathrm{mg} / \mathrm{kg}(\mathrm{n}=6)$
$\rightarrow$ Cohort $5,30 \mathrm{mg} / \mathrm{kg}(\mathrm{n}=6)$

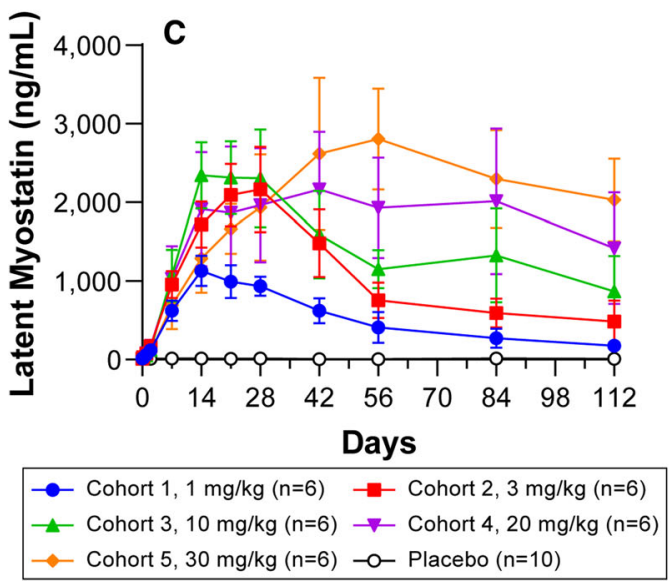

Fig. 2 Pharmacokinetic and pharmacodynamic results. a Following single ascending doses, the $C_{\max }$ for apitegromab occurred soon after completion of the IV infusion. Mean serum concentrations, $C_{\max }$, and AUC values increased proportionally with increasing dose. $\mathbf{b}$ Multiple ascending doses resulted in dose-related increases in $C_{\max }$. An accumulation of apitegromab from the first dose to the last resulted in increasing AUC values. Pre-dose time points for all subjects and all time points for placebo subjects were measured for apitegromab exposure and were reported below the limit of quantitation (BLQ). c Single

that the frequency of administration could extend beyond the 2-week dosing interval studied here, and could likely extend to monthly or longer dosing, which could represent a significant reduction of SMA patient burden. Overall, these data indicate that apitegromab achieves appropriate pharmacokinetics

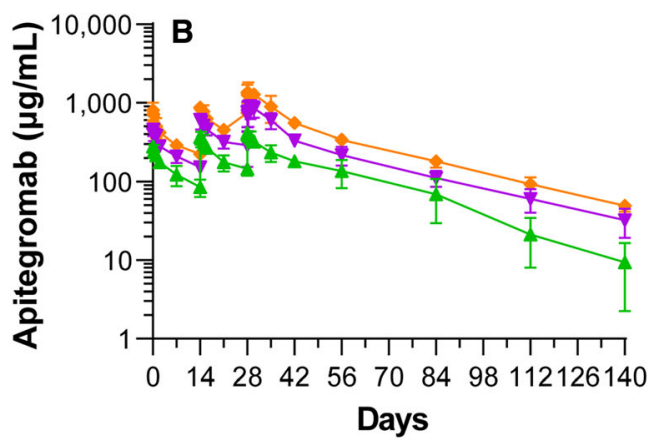

$\rightarrow$ Cohort $6,10 \mathrm{mg} / \mathrm{kg}(\mathrm{n}=8) \rightarrow-$ Cohort $7,20 \mathrm{mg} / \mathrm{kg}(\mathrm{n}=6)$ $\checkmark$ Cohort $8,30 \mathrm{mg} / \mathrm{kg}(\mathrm{n}=6)$

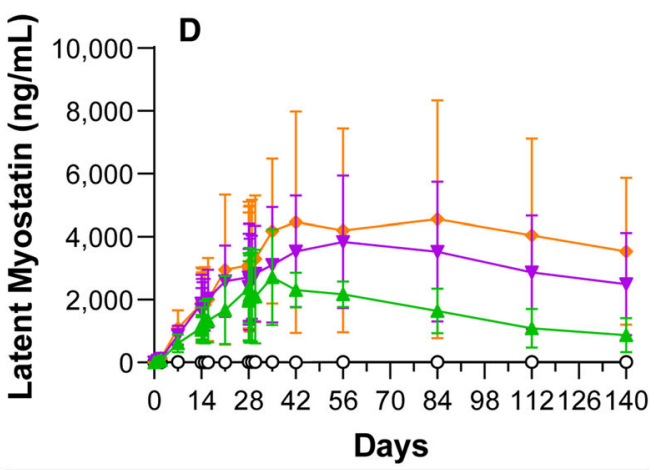

- Cohort 6, $10 \mathrm{mg} / \mathrm{kg}(\mathrm{n}=8) \rightarrow-$ Cohort $7,20 \mathrm{mg} / \mathrm{kg}(\mathrm{n}=6)$ - Cohort 8, $30 \mathrm{mg} / \mathrm{kg}(\mathrm{n}=6)$ - - Placebo $(\mathrm{n}=6)$

ascending doses of apitegromab resulted in dose-related increases in serum latent myostatin reaching a plateau between days 14 and 28 for doses $\leq 10 \mathrm{mg} / \mathrm{kg}$ and days 42 and 56 for $20 \mathrm{mg} / \mathrm{kg}$ and $30 \mathrm{mg} / \mathrm{kg}$ doses which then declined in all dose groups. $\mathbf{d}$ Following multiple ascending doses of apitegromab, mean latent myostatin concentrations increased after the first dose and remained elevated after the administration of the third dose. Latent myostatin concentrations began to decline by day 56 in the 10 and $20 \mathrm{mg} / \mathrm{kg}$ dose cohorts and day 84 in the $30 \mathrm{mg} / \mathrm{kg}$ dose cohorts

to support further studies within these dose ranges.

Serum latent myostatin was measured as an exploratory biomarker to assess the extent of target engagement across the study. While apitegromab binds to and engages both proand latent forms of myostatin with similar affinity (2.9 nM, and $2.4 \mathrm{nM}$, respectively), only 
Table 9 Summary of noncompartmental PK parameters for apitegromab by treatment: part B, multiple ascending dose

\begin{tabular}{|c|c|c|c|}
\hline Noncompartmental PK parameter & $\begin{array}{l}\text { Cohort } 6 \\
10 \mathrm{mg} / \mathrm{kg}\end{array}$ & $\begin{array}{l}\text { Cohort } 7 \\
20 \mathrm{mg} / \mathrm{kg}\end{array}$ & $\begin{array}{l}\text { Cohort } 8 \\
30 \mathrm{mg} / \mathrm{kg}\end{array}$ \\
\hline \multicolumn{4}{|l|}{ Day 0 -day 14} \\
\hline & $n=8$ & $n=6$ & $n=6$ \\
\hline \multirow[t]{2}{*}{ Mean $\mathrm{AUC}_{(0-\text { last })}, \mathrm{h}^{*} \mu \mathrm{g} / \mathrm{mL}(\mathrm{CV} \%)$} & $50,822.6(22.6)$ & $76,963.9(12.9)$ & $120,049.4(27.4)$ \\
\hline & $n=6$ & $n=6$ & $n=6$ \\
\hline Mean $\operatorname{AUC}_{(0-336)}, \mathrm{h}^{*} \mu \mathrm{g} / \mathrm{mL}(\mathrm{CV} \%)$ & $43,756.7(14.8)$ & $76,986.1(12.9)$ & $111,756.8(15.2)$ \\
\hline Dose normalized $\mathrm{AUC}_{(0-336)},\left[\mathrm{h}^{*} \mu \mathrm{g} / \mathrm{mL}\right] /[\mathrm{mg} / \mathrm{kg}]$ & 4375.7 & 3849.3 & 3725.2 \\
\hline Mean $C_{\max }, \mu \mathrm{g} / \mathrm{mL}(\mathrm{CV} \%)$ & $288.13(14.1)$ & $463(16.6)$ & $803.33(24.5)$ \\
\hline Dose normalized $C_{\max },\left[\mathrm{h}^{*} \mu \mathrm{g} / \mathrm{mL}\right] /[\mathrm{mg} / \mathrm{kg}]$ & 28.8 & 23.2 & 26.8 \\
\hline Mean $C_{\text {last }}, \mu \mathrm{g} / \mathrm{mL}(\mathrm{CV} \%)$ & $78.94(29.3)$ & $153.67(11.9)$ & $231.67(14.9)$ \\
\hline Dose normalized $C_{\text {last }},\left[\mathrm{h}^{*} \mu \mathrm{g} / \mathrm{mL}\right] /[\mathrm{mg} / \mathrm{kg}]$ & 7.9 & 7.7 & 7.7 \\
\hline Mean CL, mL/h (CV\%) & $9.823(45.1)$ & $10.852(18.5)$ & $10.714(34.9)$ \\
\hline Mean $V_{\mathrm{z}}, \mathrm{L}(\mathrm{CV} \%)$ & $4.305(18.4)$ & $4.957(9.8)$ & $5.507(10.9)$ \\
\hline \multicolumn{4}{|l|}{ Day 14-day 28} \\
\hline Mean $C_{\max }, \mu \mathrm{g} / \mathrm{mL}(\mathrm{CV} \%)$ & $390.83(18.2)$ & $650.17(14.4)$ & $934(14.1)$ \\
\hline Dose normalized $C_{\max },\left[\mathrm{h}^{*} \mu \mathrm{g} / \mathrm{mL}\right] /[\mathrm{mg} / \mathrm{kg}]$ & 39.1 & 32.5 & 31.1 \\
\hline Mean $C_{\text {last }}, \mu \mathrm{g} / \mathrm{mL}(\mathrm{CV} \%)$ & $146.33(18.2)$ & $290.67(47)$ & $743.4(33.7)$ \\
\hline Dose normalized $C_{\text {last }},\left[\mathrm{h}^{*} \mu \mathrm{g} / \mathrm{mL}\right] /[\mathrm{mg} / \mathrm{kg}]$ & 14.6 & 14.5 & 24.8 \\
\hline \multicolumn{4}{|l|}{ Day 28-study end } \\
\hline & $n=6$ & $n=6$ & $n=5$ \\
\hline \multirow[t]{2}{*}{ Mean $\mathrm{AUC}_{(0-\mathrm{last})}, \mathrm{h}^{*} \mu \mathrm{g} / \mathrm{mL}(\mathrm{CV} \%)$} & $202,085.4(45.6)$ & $488,068.3(16.9)$ & $759,224.5(12.4)$ \\
\hline & $n=5$ & $n=6$ & $n=5$ \\
\hline Mean $\operatorname{AUC}_{(0-336)}, h^{*} \mu \mathrm{g} / \mathrm{mL}(\mathrm{CV} \%)$ & $83,902.7(17.6)$ & $201,619.1(15.1)$ & $305,647(16.8)$ \\
\hline Dose normalized $\mathrm{AUC}_{(0-336)},\left[\mathrm{h}^{*} \mu \mathrm{g} / \mathrm{mL}\right] /[\mathrm{mg} / \mathrm{kg}]$ & 8390.3 & $10,081.0$ & $10,188.2$ \\
\hline Median $T_{\max }, \mathrm{h}(\min , \max )$ & $2.02(2,6)$ & $37.99(2,50)$ & $10(6,50)$ \\
\hline Mean $C_{\max }, \mu \mathrm{g} / \mathrm{mL}(\mathrm{CV} \%)$ & $421(15.2)$ & $958.67(17.1)$ & $1582(20)$ \\
\hline Dose normalized $C_{\max },\left[\mathrm{h}^{*} \mu \mathrm{g} / \mathrm{mL}\right] /[\mathrm{mg} / \mathrm{kg}]$ & 42.1 & 47.9 & 52.7 \\
\hline Mean $\left.C_{\text {last }}, \mu \mathrm{g} / \mathrm{mL} \mathrm{CV} \%\right)$ & $66.25(147.4)$ & $32.3(39.8)$ & $49.4(15.9)$ \\
\hline Dose normalized $C_{\mathrm{last}},\left[\mathrm{h}^{*} \mu \mathrm{g} / \mathrm{mL}\right] /[\mathrm{mg} / \mathrm{kg}]$ & 6.6 & 1.6 & 1.6 \\
\hline Mean $\mathrm{RC}_{\max }, \mu \mathrm{g} / \mathrm{mL}(\mathrm{CV} \%)$ & $1.419(3.6)$ & $2.119(22.9)$ & $2.117(16.3)$ \\
\hline Mean $\operatorname{RAUC}_{(0-336)}, \mu \mathrm{g} / \mathrm{mL}(\mathrm{CV} \%)$ & $1.879(13.4)$ & $2.637(13.7)$ & $2.851(14.2)$ \\
\hline Mean CL, mL/h (CV\%) & $10.263(28.6)$ & $8.19(19.7)$ & $8.332(24.4)$ \\
\hline
\end{tabular}


Table 9 continued

\begin{tabular}{llll}
\hline Noncompartmental PK parameter & $\begin{array}{l}\text { Cohort } \mathbf{6} \\
\mathbf{1 0 ~} \mathbf{~ g} / \mathbf{k g}\end{array}$ & $\begin{array}{l}\text { Cohort 7 } \\
\mathbf{2 0} \mathbf{~ m g} / \mathbf{k g}\end{array}$ & $\begin{array}{l}\text { Cohort 8 } \\
\mathbf{3 0} \mathbf{~ m g} / \mathbf{k g}\end{array}$ \\
\hline Mean $V_{\mathrm{z}}, \mathrm{L}(\mathrm{CV} \%)$ & $7.224(20.7)$ & $8.691(29.8)$ & $8.501(19.7)$ \\
\hline
\end{tabular}

$A U C_{(0-336)}$ area under the concentration-time curve from time 0 to $336 \mathrm{~h}, A U C_{(0-\text { last })}$ area under the concentration-time curve to the last measurable concentration, $C_{\text {last }}$ last measurable concentration, $C_{\max }$ maximum serum concentration, $C L$ total body clearance, $C V$ coefficient of variation, max maximum, min minimum, $R A U C_{(0-336)}$ accumulation ratio based on area under the concentration-time curve from 0 to $336 \mathrm{~h}, R C_{\max }$ accumulation ratio based on maximum concentrations, $P K$ pharmacokinetics, $S A D$ single ascending dose, $t_{1 / 2}$ terminal elimination half-life, $T_{\text {max }}$ time to maximum serum concentration, $V_{z}$ volume of distribution

latent myostatin is detected in the serum [10]. The bioanalytical assay used to measure serum latent myostatin implements an acid-dissociation step to dissociate the drug-antigen complex, allowing for the quantitation of total serum latent myostatin [25]. In part A (SAD) of the study, serum latent myostatin levels increased and peaked at about 2000-3000 ng/ $\mathrm{mL}$ by days $14-28$. Latent myostatin levels only reached approximately half of this maximal value at the lowest dose tested $(1 \mathrm{mg} / \mathrm{kg})$, before returning to baseline. At all other doses, after reaching this maximal latent myostatin accumulation, latent myostatin remained elevated for a period of time before beginning to return to baseline levels. In part B (MAD) of the study, latent myostatin levels increased after the first dose and remained elevated after administration of the third dose. Latent myostatin concentrations began to decline by day 56 in the 10 and $20 \mathrm{mg} / \mathrm{kg}$ dose cohorts and day 84 in the $30 \mathrm{mg} / \mathrm{kg}$ dose cohorts. These increases of latent myostatin in serum indicate apitegromab has robust target engagement.

This phenomenon occurs with inhibition of serum cytokines [26], and is assumed to be due to the long half-life of the drug extending the half-life of the antigen. As latent myostatin is bound by apitegromab in muscle, it is likely that this inactive drug-antigen complex is flushed into the bloodstream and degraded by typical pathways. Since apitegromab has a long half-life, serum latent myostatin remains engaged until drug is sufficiently cleared from circulation. This relationship suggests that apitegromab doses at above $3 \mathrm{mg} / \mathrm{kg}$ allow for full engagement of latent myostatin, and higher doses allow for longer duration of full target engagement. These results are consistent with trends seen in both cynomolgus monkeys treated with apitegromab [25] and in an SMN $\Delta 7$ mouse model of SMA after treatment with the parental muSRK-015P molecule [21].

On the basis of its AE profile, apitegromab appears to be safe and well tolerated, with no clinically meaningful changes in baseline vital signs, ECGs, or clinical laboratory parameters and no ADA formation. The primary and secondary objectives of this study support the safety and tolerability of apitegromab at doses up to $30 \mathrm{mg} / \mathrm{kg}$ by IV infusion. These data, along with available non-clinical data, support further investigation of apitegromab in human subjects.

Apitegromab is currently under investigation in a 52-week phase 2 clinical study (NCT03921528). On the basis of the results of the current study, apitegromab is dosed via intravenous infusion every 4 weeks, as either monotherapy or as an additive therapy to SMN upregulators, to assess possible improvements in downstream muscular function in patients with later-onset SMA (types 2 and 3, aged 2-21 years) [27]. Two doses of apitegromab, $2 \mathrm{mg} /$ $\mathrm{kg}$ and $20 \mathrm{mg} / \mathrm{kg}$, were selected on the basis of its well-characterized PK and informative PD (target engagement) profiles revealed in healthy subjects. Ultimately, the results of the phase 2 clinical study will offer a better understanding of PK/PD features of apitegromab, the relationship of apitegromab concentrations and changes in latent myostatin in the serum to clinical 
efficacy and safety outcomes, and ultimately the value of measuring latent myostatin concentrations in the serum as a biomarker of target engagement in patients with SMA.

Study limitations include evaluation of safety, tolerability, and PK/PD in an adult healthy volunteers-only study, which is typical in first-in-human phase 1 studies. Evaluation of safety, tolerability, and PK/PD responses in children with the targeted clinical indication usually occurs in a phase 2 exploratory trial that is currently ongoing. Neither muscle-specific effects nor the impact on growth or related hormonal changes, and development milestones were assessed in this first-in-human phase 1 study. The primary objective of the phase 1 study was to evaluate the safety and tolerability of single and multiple apitegromab doses up to $30 \mathrm{mg} / \mathrm{kg}$ by IV infusion to healthy subjects. The secondary objectives were to evaluate the PK, PD, and immunogenicity of single and multiple apitegromab doses up to $30 \mathrm{mg} / \mathrm{kg}$ by IV infusion to healthy subjects.

\section{CONCLUSIONS}

Apitegromab (SRK-015) is an investigational, fully human, monoclonal antibody that specifically binds to proforms of myostatin, which include promyostatin and latent myostatin, inhibiting myostatin activation. The results of this phase 1 double-blind, placebo-controlled study demonstrate that apitegromab has a mean apparent terminal half-life of 24-31 days after single intravenous doses and dose-related increases in $C_{\max }$ following multiple ascending doses in healthy volunteers. Both single and multiple doses of apitegromab resulted in dosedependent and sustained increases in serum latent myostatin, indicating robust target engagement. Apitegromab was safe and well tolerated. These results support ongoing development of this molecule.

\section{ACKNOWLEDGEMENTS}

The authors gratefully thank the subjects of this study for their participation.
Funding. This study was sponsored by Scholar Rock, Inc., Cambridge, MA. Rapid Service and Open Access Fees were paid by Scholar Rock.

Editorial Assistance. The authors acknowledge the editorial assistance of Carl S. Hornfeldt, PhD, Apothekon, Inc., during the preparation of this manuscript with funding provided by Scholar Rock, Inc..

Authorship. All named authors meet the International Committee of Medical Journal Editors (ICMJE) criteria for authorship for this article, take responsibility for the integrity of the work as a whole, and have given their approval for this version to be published.

Authors' Contributions. Analyzed the data [Doreen Barrett, Ryan Iarrobino, Sanela Bilic, Shaun M. Cote, Katherine Kacena, Yung Chyung]. Design of the study [Doreen Barrett, Ryan Iarrobino, Ashish Kalra, Sanela Bilic, Shaun M. Cote, Katherine Kacena, Yung Chyung, James Gordon Still]. Interpreted the data [Doreen Barrett, George Nomikos, Ryan Iarrobino, Ashish Kalra, Sanela Bilic, Shaun M. Cote, Katherine Kacena, Yung Chyung, James Gordon Still]. Major role in acquisition of data [Shaun M. Cote, Leela Vrishabhendra]. Performed Research [Shaun M. Cote]. Revised the manuscript for intellectual content [Doreen Barrett, George Nomikos, Ryan Iarrobino, Amy Place, Ashish Kalra, Sanela Bilic, Shaun M. Cote, Kimberly Long]. Wrote manuscript [Amy Place, Sanela Bilic, Shaun M. Cote].

Prior Presentation. The results of this study were presented, in part, at the 24th International Annual Congress of the World Muscle Society, Copenhagen Denmark, October 1-15, 2019; SMA Europe 2nd International SMA Research Congress, Evry France, February 5-7, 2020; Cure SMA Virtual Annual Conference, June 8-12, 2020; 25th International Annual Virtual Congress of the World Muscle Society, September 28 to October 2, 2020.

Disclosures. Doreen Barrett, Yung Chyung, Shaun M. Cote, Ryan Iarrobino, Ashish Kalra, 
Kimberly Long, George Nomikos, and Amy Place are employees of Scholar Rock, Inc., and own equity in the company. Sanela Bilic, Katherine Kacena, James Gordon Still, and Leela Vrishabhendra were paid consultants. Current address for Kimberly Long, PhD is Casma Therapeutics, Inc., 400 Technology Square Suite 201, Cambridge, MA 02139.

Compliance with Ethics Guidelines. The protocol and all protocol amendments were signed and dated by the investigator and approved in writing by a commercial institutional review board (Advarra ${ }^{\circledR}$, Columbia, MD, Ref 00024966) in accordance with Good Clinical Practice (GCP) prior to implementation. In addition, the IRB approved the written informed consent form, including the ability to publish data from the study, any consent form updates, subject recruitment procedures (e.g., advertisements), and any written information provided to the subjects prior to implementation. All subjects provided informed written consent to participate in the study prior to any study-related activities.

Data Availability. All data generated or analyzed during this study are included in this published article.

Open Access. This article is licensed under a Creative Commons Attribution-NonCommercial 4.0 International License, which permits any non-commercial use, sharing, adaptation, distribution and reproduction in any medium or format, as long as you give appropriate credit to the original author(s) and the source, provide a link to the Creative Commons licence, and indicate if changes were made. The images or other third party material in this article are included in the article's Creative Commons licence, unless indicated otherwise in a credit line to the material. If material is not included in the article's Creative Commons licence and your intended use is not permitted by statutory regulation or exceeds the permitted use, you will need to obtain permission directly from the copyright holder. To view a copy of this licence, visit http://creativecommons.org/licenses/by$\mathrm{nc} / 4.0 /$.

\section{REFERENCES}

1. Arnold WD, Kassar D, Kissel JT. Spinal muscular atrophy: diagnosis and management in a new therapeutic era. Muscle Nerve. 2015;51:157-67.

2. Monani UR, Lorson CL, Parsons DW, et al. A single nucleotide difference that alters splicing patterns distinguishes the SMA gene SMN1 from the copy gene SMN2. Hum Mol Genet. 1999;8:1177-83.

3. Lefebvre S, Bürglen L, Reboullet S, et al. Identification and characterization of a spinal muscular atrophy-determining gene. Cell. 1995;80:155-65.

4. Kolb SJ, Kissel JT. Spinal muscular atrophy. Neurol Clin. 2015;33:831-46.

5. Health and Resources and Services Administration. Newborn screening for spinal muscular atrophy. A summary of the evidence and advisory committee decision. March 13, 2018. https://www.hrsa.gov/ sites/default/files/hrsa/advisory-committees/heritab le-disorders/rusp/previous-nominations/sma-consu mer-summary.pdf. Accessed Jul 2020.

6. Chen TH. New and developing therapies in spinal muscular atrophy: from genotype to phenotype to treatment and where do we stand? Int J Mol Sci. 2020;21:3297.

7. Audic F, de la Banda MGG, Bernoux D, RamirezGarcia P, Durigneux J. Effects of nusinersen after one year of treatment in 123 children with SMA type 1 or 2: a French real-life observational study. Orphanet J Rare Dis. 2020;15:148.

8. Dabbous O, Maru B, Jansen JP, et al. Survival, motor function, and motor milestones: comparison of AVXS-101 relative to nusinersen for the treatment of infants with spinal muscular atrophy type 1 . Adv Ther. 2019;36:1164-76.

9. Hensel N, Kubinski S, Claus P. The need for SMNindependent treatments of spinal muscular atrophy (SMA) to complement SMN-enhancing drugs. Front Neurol. 2020;11:45.

10. Pirruccello-Straub M, Jackson J, Wawersik S, et al. Blocking extracellular activation of myostatin as a strategy for treating muscle wasting. Sci Rep. 2018;8:2292.

11. Grobet L, Martin LJ, Poncelet D, et al. A deletion in the bovine myostatin gene causes the double-muscled phenotype in cattle. Nat Genet. 1997;17:71-4.

12. Mosher DS, Quignon P, Bustamante CD, et al. A mutation in the myostatin gene increases muscle mass and enhances racing performance in heterozygote dogs. PLoS Genet. 2007;3:e79. 
13. Schuelke M, Wagner KR, Stolz LE, et al. Myostatin mutation associated with gross muscle hypertrophy in a child. N Engl J Med. 2004;350:2682-8.

14. Han HQ, Zhou X, Mitch WE, Goldberg AL. Myostatin/activin pathway antagonism: molecular basis and therapeutic potential. Int J Biochem Cell Biol. 2013;45:2333-47.

15. Wagner KR, Fleckenstein JL, Amato AA, et al. A phase I/II trial of MYO-029 in adult subjects with muscular dystrophy. Ann Neurol. 2008;63:561-71.

16. Becker C, Lord SR, Studenski SA, et al. Myostatin antibody (LY2495655) in older weak fallers: a proofof-concept, randomised, phase 2 trial. Lancet Diabetes Endocrinol. 2015;3:948-57.

17. Golan T, Geva R, Richards D, et al. LY2495655, an antimyostatin antibody, in pancreatic cancer: a randomized, phase 2 trial. J Cachexia Sarcopenia Muscle. 2018;9:871-9.

18. Garito T, Zakaria M, Papanicolaou DA, et al. Effects of bimagrumab, an activin receptor type II inhibitor, on pituitary neurohormonal axes. Clin Endocrinol (Oxf). 2018;88:908-19.

19. Campbell C, McMillan HJ, Mah JK, et al. Myostatin inhibitor ACE-031 treatment of ambulatory boys with Duchenne muscular dystrophy: results of a randomized, placebo-controlled clinical trial. Muscle Nerve. 2017;55:458-64.

20. Anderson SB, Goldberg AL, Whitman M. Identification of a novel pool of extracellular pro-myostatin in skeletal muscle. J Biol Chem. 2008;283: 7027-35.

21. Long KK, O'Shea KM, Khairallah RJ, et al. Specific inhibition of myostatin activation is beneficial in mouse models of SMA therapy. Hum Mol Genet. 2019;28:1076-89.

22. National Cancer Institute. Common Terminology Criteria for Adverse Events (CTCAE) Version 5.0, November 27, 2017. U.S. Department of Health and Human Services. https://ctep.cancer.gov/protocold evelopment/electronic_applications/docs/CTCAE_ v5_Quick_Reference_5x7.pdf. Accessed Jul 2020.

23. Bhattacharya I, Pawlak S, Marraffino S, et al. Safety, tolerability, pharmacokinetics, and pharmacodynamics of domagrozumab (PF-06252616), an antimyostatin monoclonal antibody, in healthy subjects. Clin Pharmacol Drug Dev. 2018;7:484-97.

24. Glasser CE, Gartner MR, Wilson D, Miller B, Sherman ML, Attie K. Locally acting ACE-083 increases muscle volume in healthy volunteers. Muscle Nerve. 2018;57:921-6.

25. Cote CM, Jackson J, Pirruccello-Straub M, Carven GJ, Wawersik S. A sensitive and selective immunoassay for the quantitation of serum latent myostatin after in vivo administration of SRK-015, a selective inhibitor of myostatin activation. SLAS Discov. 2020;25:95-103.

26. Klein B, Brailly H. Cytokine-binding proteins: stimulating antagonists. Immunol Today. 1995;16: 216-20.

27. Place A, Scholar Rock SRK-015 Team. A phase 2 study to evaluate the efficacy and safety of SRK-015 in patients with later-onset spinal muscular atrophy (TOPAZ): an introduction. Presented at the 2nd International Scientific \& Clinical Congress on Spinal Muscular Atrophy. Evry, France, February 5-7, 2020. 